\title{
Association of Sarcopenic Obesity and Body Composition With Metabolically Unhealthy Overweight/Obese Phenotypes Among Iranian Women: A Cross-Sectional Study
}

\author{
Aliyu Tijani Jibril \\ Tehran University of Medical Sciences \\ Atieh Mirzababaei \\ Tehran University of Medical Sciences \\ Farideh Shiraseb \\ Tehran University of Medical Sciences \\ Niloufar Rasaei \\ Tehran University of Medical Sciences \\ Khadijeh Mirzaei ( $\square$ mina_mirzaei101@yahoo.com ) \\ Tehran University of Medical Sciences https://orcid.org/0000-0002-7554-8551
}

\section{Research note}

Keywords: Sarcopenic obesity, body composition, metabolic healthy obesity, metabolic unhealthy obesity

Posted Date: February 2nd, 2021

DOl: https://doi.org/10.21203/rs.3.rs-154683/v1

License: (c) (1) This work is licensed under a Creative Commons Attribution 4.0 International License.

Read Full License 


\section{Abstract \\ Objectives}

Obesity is a major risk factor for metabolic syndrome, with its prevalence has increased over the past decade. Major changes in body composition with aging have a significant effect on many clinical outcomes. Sarcopenic obesity consists of both the presence of abnormal adipose tissue with a deficit of muscle mass.

\section{Results}

Of the 241 subjects in this study (average age 35.32 years), 176 (73.03\%) were classified as MUO phenotype. Based on this study, the prevalence of sarcopenic obesity was $7.88 \%$. We found that high fatfree mass was more strongly and significantly associated with MUO phenotype. Furthermore, we found that individuals with high fat-free mass and high skeletal muscle mass had a significantly low prevalence of MUO phenotype. A significant positive correlation between metabolic phenotypes and sarcopenic obesity was also observed after all potential covariates were adjusted for. These results of this study suggest that increased adiposity and decreased skeletal muscle mass are associated with unfavorable metabolic traits among overweight and obese Iranian women. SO was also found to be associated with a greater risk of developing MUO phenotype.

\section{Introduction}

Obesity is an abnormal accumulation of fat that affects health negatively [1] with its prevalence having doubled since 1980 in middle-aged and older adults [2]. Previous studies reported $16.5 \%$ of Iranian subjects older than 18 years [3] and $24.8 \%$ of women aged 30 years and above as obese [4]. Obese subjects have been reported to have heterogeneous phenotypes with different degrees of metabolic risk [5]. A sub-group of obese individuals without associated metabolic complications have been phenotypically described as metabolically healthy obese $(\mathrm{MHO})$ individuals $[6,7]$. On the other hand is the metabolically unhealthy obese (MUO) individuals characterized by obesity-related metabolic complications [8]. Previous studies have reported MHO phenotype to be associated with a healthy metabolic profile, characterized by a lower amount of visceral adipose tissue (VAT) and liver fat [9], favorable lipid profile, high insulin sensitivity, and low pro-inflammatory cytokine levels in plasma $[9,10]$ than MUO subjects.

Major changes associated with aging in body composition include a decline in both fat-free mass and muscle strength referred to as sarcopenia [11]; and, an increase in body fat and a decline in skeletal muscle referred to as sarcopenic obesity (SO) [12]. Numerous studies have reported a significantly higher risk of mortality [13] worse cardiovascular risk profiles including hyperglycemia, hypertension, dyslipidemia, insulin resistance, and lower cardiorespiratory fitness [14-16] in sarcopenic obese individuals compared to non-sarcopenic or non-obese subjects. 
Previous reports indicate a 9.9\% prevalence of SO among Tehranian overweight/obese women [17], while a $10.9 \%$ and a $7.2 \%$ prevalence of $\mathrm{MUO}$ and $\mathrm{MHO}$ phenotypes respectively in Iranian adult population [18]. The aim of this study therefore was to determine the association of sarcopenic obesity and body composition with metabolically unhealthy overweight/obese phenotypes among Iranian women.

\section{Methods}

\section{Study design and Population}

This cross-sectional study was conducted among 241 women referred to health centers affiliated to Tehran University Medical Science (TUMS) in Iran. Subjects were registered by the use of multistage cluster random sampling method. Inclusion criteria were age 18-48 years and being overweight/obese. Exclusion criteria included having; an acute or chronic inflammatory disease, regular use of medication, history of hypertension, cardiovascular disease, diabetes mellitus, impaired renal and liver function, intake of alcohol or drug abuse, smoking, thyroid disease, malignancies, pregnancy, and lactation. All participants were informed about the study aims and procedures and provided written informed consent. The Medical Research Ethics Committee of TUMS approved the study with the following identification IR.TUMS.VCR.REC.1398.692.

\section{Measurements}

\section{Dietary Assessment}

A semi-quantitative food frequency questionnaire (FFQ) with 147 food items was applied to evaluate the usual dietary intake. Predictable average daily intakes of food parameters were computed using NUTRITIONIST IV software (version 7.0; N-Squared Computing, Salem, OR), modified for Iranian foods.

\section{Measurement of blood samples}

Blood samples were collected following overnight fasting. Fasting blood sugar, triglyceride, total cholesterol, high-density lipoprotein (HDL), low-density lipoprotein (LDL), and high-sensitive C-reactive protein (hs-CRP) were assessed using a package from Randox Laboratories (Hitachi 902).

\section{Body composition and anthropometric measurements assessment}

Bioelectrical impedance analyzer (BIA) (InBody 720, South Korea) was used to assess body composition indicators, including body fat-free mass and fat mass. Anthropometric measurements such as weight, height, waist circumference, and hip circumference were performed on all participants. BMI was then calculated by dividing the weight $(\mathrm{kg})$ by the square of the height $(\mathrm{m})$.

\section{Assessment of blood pressure}


The blood pressure was assessed after the participants rested for 10 minutes. Hypertension was defined as blood pressure $>130 / 85 \mathrm{mmHg}$. This category includes patients taking antihypertensive medicines, even if treatment achieves a blood pressure level that is within the target range.

\section{The HOMA-IR calculation}

The insulin resistance homeostatic model assessment (HOMA-IR) was based on the following equation: [fasting plasma glucose $(\mathrm{mmol} / \mathrm{l}) \times$ fasting plasma insulin $(\mathrm{mlU} / \mathrm{l})] / 22.5$ [19].

\section{Definition of metabolic health and sarcopenic obesity}

The Karelis criteria were used because all inflammation criteria are tested. The existence of four or more of the following five is described as metabolic abnormality according to the requirements of Karelis, composition: TG $1.7 \mathrm{mmol} / \mathrm{l}, \mathrm{HDL} 1.3 \mathrm{mmol} / \mathrm{l}$, without treatment; LDL $2.6 \mathrm{mmol} / \mathrm{l}$, without treatment, $3.0 \mathrm{mg} / \mathrm{l} \mathrm{hs}$-CRP and 2.7 HOMA-IR [20]. We assessed SO as two lower quintiles of SMM and two highest quintiles of FM [21] using BIA.

\section{Other baseline measurements}

The socio-demographic characteristics assessed by questionnaire included age, gender, educational level attained, physical activity status, and smoking habits. The validated International Physical Activity Questionnaire (short form) was used to assess the level of physical activity.

\section{Statistical Analysis}

All statistical analyses were performed using the IBM SPSS software version 25.0 (SPSS, Chicago, IL, USA), and $p$-values less than 0.05 were considered statistically significant. Normal distribution of data was checked by the Kolmogorov-Smirnov test. Continuous variables were represented as mean \pm standard deviations (SDs), and categorical variables represented as percentages and numbers. An independent sample t-test (continuous variables) and a chi-square $\left(\chi^{2}\right)$ tests (categorical variables) were used to assess differences between the study groups. Spearman bivariate correlation between body composition variables and sarcopenic obesity was performed. Association between metabolic healthy statuses with measures of body composition and sarcopenic obesity were assessed using Binary logistic regression.

Results

\section{General characteristics of study participants}

The general characteristics of the study subjects are shown in Table 1. In total, 241 women (35.32 \pm 8.68 years) participated in our study. The prevalence of SO was $7.88 \%$. Subjects in the MUO group had a significantly higher body weight, BMI, WC, BFM, and VFA compared to subjects in the MHO group ( $p<$ $0.001)$. The FFM $(p=0.004), \operatorname{FMI}(p=0.001)$, and SMM $(p=0.002)$ were significantly higher in the MUO group. TG, LDL, and total cholesterol were significantly higher $(p<0.001)$ in the unhealthy group, while the levels of HDL were significantly higher $(p<0.001)$ in the healthy group. A significant difference was also 
observed among the groups base on HOMA-IR $(p<0.001)$ and insulin level $(p=0.001)$. A significantly higher diastolic blood pressure and mean hs-CRP level were observed among the unhealthy group $(p<$ $0.001)$. 
Table 1

General characteristics of study participants

\begin{tabular}{lllll} 
variables & $\begin{array}{l}\text { Metabolically } \\
\text { Healthy }\end{array}$ & $\begin{array}{l}\text { Metabolically } \\
\text { Unhealthy }\end{array}$ & $\begin{array}{l}\text { P- } \\
\text { value }^{*}\end{array}$ & $\begin{array}{l}\text { P- } \\
\text { value }^{* * *}\end{array}$ \\
\cline { 2 - 3 } & &
\end{tabular}

Demographic characteristic

$\begin{array}{lllll}\text { Age (year) }^{a} & 35.92 \pm 7.55 & 36.32 \pm 8.68 & 0.74 & 0.84 \\ \text { Marital status }^{b} & 17(34.0) & 33(66.0) & 0.21 & 0.80 \\ \text { Single (\%) } & 47(25.0) & 141(75.0) & & \end{array}$

Married (\%)

$\begin{array}{lllll}\text { Education }^{\mathrm{b}} & 1(33.3) & 2(66.7) & 0.57 & 0.73 \\ \text { Illiterate (\%) } & 4(16.0) & 21(84.0) & & \\ \text { Under diploma (\%) } & 24(26.1) & 68(73.9) & \\ \text { Diploma (\%) } & 35(27.7) & 83(70.3) & \end{array}$

Bachelor and above (\%)

$\begin{array}{lllll}\text { Economic status }^{\text {b }} & 6(19.4) & 25(80.6) & 0.02 & 0.16 \\ \text { Low class }(\%) & 20(48.8) & 21(51.2) & & \\ \text { Middle class }(\%) & 7(29.2) & 17(70.8) & \\ \text { High class }(\%) & & & \end{array}$

$\begin{array}{lllll}\text { Sarcopenic obesity }^{\mathrm{b}} & 61(27.5) & 161(72.5) & 0.06 & 0.04\end{array}$

Non-sarcopenic obese (\%)

Sarcopenic obese (\%)

4 (21.1)

$15(78.9)$

Anthropometry and Body Composition

\begin{tabular}{|c|c|c|c|c|}
\hline Weight (kg) ${ }^{a}$ & $74.89 \pm 8.71$ & $81.03 \pm 11.02$ & $\begin{array}{l}<.001 \\
0\end{array}$ & $<0.001$ \\
\hline Height $(\mathrm{cm})^{a}$ & $161.09 \pm 5.18$ & $161.51 \pm 5.83$ & 0.06 & 0.92 \\
\hline $\mathrm{BMI}\left(\mathrm{kg} / \mathrm{m}^{2}\right)^{\mathrm{a}}$ & $28.75 \pm 2.86$ & $31.11 \pm 3.64$ & $\dot{0}_{0.001}$ & $<0.001$ \\
\hline $\mathrm{WC}(\mathrm{cm})^{\mathrm{a}}$ & $93.38 \pm 7.01$ & $99.64 \pm 9.27$ & $\hat{0}_{0.001}$ & $<0.001$ \\
\hline Body fat mass $(\mathrm{kg})^{\text {a }}$ & $29.76 \pm 5.96$ & $34.03 \pm 7.34$ & $<.001$ & $<0.001$ \\
\hline
\end{tabular}




\begin{tabular}{|c|c|c|c|c|}
\hline \multirow[t]{2}{*}{ variables } & $\begin{array}{l}\text { Metabolically } \\
\text { Healthy }\end{array}$ & $\begin{array}{l}\text { Metabolically } \\
\text { Unhealthy }\end{array}$ & \multirow[t]{2}{*}{$\begin{array}{l}\text { P- } \\
\text { value }^{*}\end{array}$} & \multirow[t]{2}{*}{$\begin{array}{l}\text { P- } \\
\text { value }^{\ddagger^{\star *}}\end{array}$} \\
\hline & \multicolumn{2}{|l|}{$($ mean \pm SD) } & & \\
\hline Visceral fat area $\left(\mathrm{cm}^{2}\right)^{a}$ & $14.04 \pm 2.89$ & $15.72 \pm 3.25$ & $<.001$ & 0.001 \\
\hline Fat-free mass (kg) a & $44.84 \pm 4.68$ & $47.10 \pm 5.57$ & 0.004 & 0.01 \\
\hline Skeletal muscle mass $(\mathrm{kg})^{\mathrm{a}}$ & $24.42 \pm 2.69$ & $25.89 \pm 3.32$ & 0.002 & 0.008 \\
\hline Fat free mass index $(\mathrm{kg})^{a}$ & $17.26 \pm 1.21$ & $18.75 \pm 9.88$ & 0.23 & 0.27 \\
\hline Fat mass index $(\mathrm{kg})^{a}$ & $11.65 \pm 2.62$ & $13.06 \pm 2.78$ & 0.001 & $<0.001$ \\
\hline \multicolumn{5}{|l|}{ Blood Parameters } \\
\hline FBG $(\mathrm{mg} / \mathrm{dL})^{a}$ & $4.68 \pm 0.40$ & $4.86 \pm 0.49$ & 0.05 & 0.03 \\
\hline $\mathrm{TG}(\mathrm{mg} / \mathrm{dL})^{\mathrm{a}}$ & $0.90 \pm 0.28$ & $1.52 \pm 0.82$ & $<.001$ & $<0.001$ \\
\hline $\mathrm{HDL}(\mathrm{mg} / \mathrm{dL})^{a}$ & $1.34 \pm 0.24$ & $1.14 \pm 0.26$ & $\begin{array}{l}<.001 \\
0.00\end{array}$ & $<0.001$ \\
\hline $\operatorname{LDL}(\mathrm{mg} / \mathrm{dL})^{\mathrm{a}}$ & $2.19 \pm 0.50$ & $2.53 \pm 0.62$ & $\hat{0}_{0.001}$ & 0.001 \\
\hline Total cholesterol $(\mathrm{mg} / \mathrm{dL})^{\mathrm{a}}$ & $4.37 \pm 0.74$ & $4.89 \pm 0.93$ & $\begin{array}{l}<.001 \\
0.001\end{array}$ & 0.003 \\
\hline Insulin (mIU/l) & $1.13 \pm 0.24$ & $1.24 \pm 0.22$ & 0.001 & 0.01 \\
\hline HOMA index ${ }^{a}$ & $2.47 \pm 0.85$ & $3.66 \pm 1.24$ & $\dot{0} 001$ & $<0.001$ \\
\hline \multicolumn{5}{|l|}{ Blood Pressure } \\
\hline $\operatorname{SBP}(\mathrm{mmHg})^{a}$ & $109.01 \pm 12.44$ & $112.66 \pm 13.92$ & 0.06 & 0.18 \\
\hline $\operatorname{DBP}(\mathrm{mmHg})^{a}$ & $73.74 \pm 8.29$ & $79.39 \pm 10.05$ & $<.001$ & 0.007 \\
\hline \multicolumn{5}{|c|}{ Inflammatory parameter and other variables } \\
\hline $\mathrm{Hs}-\mathrm{CRP}(\mathrm{mg} / \mathrm{L})^{a}$ & $1.33 \pm 1.18$ & $5.37 \pm 4.91$ & $<.001$ & $<0.001$ \\
\hline $\begin{array}{l}\text { Physical activity (MET } \\
\text { min/week) }\end{array}$ & $989.33 \pm 1078.53$ & $1304.14 \pm 2404.33$ & 0.33 & 0.29 \\
\hline \multicolumn{5}{|c|}{$\begin{array}{l}\text { SD, standard deviation; BMI, body mass index; WC, waist circumference; FBG, fasting blood glucose; } \\
\text { TG, triglyceride; HDL, high-density lipoprotein; LDL, low-density lipoprotein; hs-CRP, High sensitive c- } \\
\text { reactive protein; SBP, systolic blood pressure; DBP, diastolic blood pressure. }\end{array}$} \\
\hline
\end{tabular}




\begin{tabular}{|lllll|}
\hline variables & $\begin{array}{l}\text { Metabolically } \\
\text { Healthy }\end{array}$ & $\begin{array}{l}\text { Metabolically } \\
\text { Unhealthy }\end{array}$ & $\begin{array}{l}\text { P- } \\
\text { value }^{*}\end{array}$ & $\begin{array}{l}\text { P- } \\
\text { value }\end{array}$ \\
\cline { 2 - 3 } & & & \\
\hline (mean \pm SD $)$ & & & \\
\hline
\end{tabular}

Data are presented as mean \pm standard deviation (SD) or percent.

${ }^{\ddagger}$ Collinear variables did not enter into the model and this P-value obtained from ANCOVA analysis

*p-value obtained from independent T-test

** p-value obtained from ANCOVA test, variable adjust for age, physical activity, energy intake, and $\mathrm{BMI}$

Chi-square and analysis of variance were used for qualitative and quantitative variables respectively, and the $p$-value was set to $<0.05$.

a Mean \pm SD

b Sample size (\%)

\section{Dietary intake of study subjects according to metabolic statuses}

As shown in Table 2, the food groups, mean dietary intakes, and the mean nutrient intakes of the study participants were not statistically significantly different between the $\mathrm{MHO}$ and $\mathrm{MUO}$ groups even after controlling for potential confounding variables $(p>0.05)$. 
Table 2

Dietary intake of study subjects according to metabolic statuses

\begin{tabular}{|c|c|c|c|c|c|c|}
\hline \multirow[t]{2}{*}{ Variables } & \multicolumn{2}{|c|}{ Metabolic healthy } & \multicolumn{2}{|c|}{ Metabolic unhealthy } & \multirow[t]{2}{*}{ P-value ${ }^{*}$} & \multirow[t]{2}{*}{ P-value $e^{* \star}$} \\
\hline & \multicolumn{2}{|l|}{ Mean SD } & \multicolumn{2}{|l|}{ Mean SD } & & \\
\hline \multicolumn{7}{|l|}{ Food groups } \\
\hline Fruits $(\mathrm{g} / \mathrm{d})$ & 508.45 & 373.30 & 505.83 & 352.66 & 0.96 & 0.34 \\
\hline $\operatorname{Vegetables}(\mathrm{g} / \mathrm{d})$ & 379.99 & 227.11 & 387.94 & 248.29 & 0.82 & 0.12 \\
\hline Cereal(g/d) & 422.18 & 195.27 & 427.86 & 226.56 & 0.86 & 0.61 \\
\hline Whole grains $(g / d)$ & 58.64 & 53.41 & 65.77 & 62.43 & 0.42 & 0.31 \\
\hline Refined grains(g/d) & 363.54 & 203.95 & 362.45 & 211.23 & 0.97 & 0.43 \\
\hline $\operatorname{Nuts}(g / d)$ & 15.23 & 16.91 & 19.06 & 28.03 & 0.96 & 0.67 \\
\hline Legumes $(\mathrm{g} / \mathrm{d})$ & 51.64 & 55.38 & 48.23 & 34.54 & 0.57 & 0.62 \\
\hline Red meat $(\mathrm{g} / \mathrm{d})$ & 21.61 & 17.70 & 22.53 & 19.75 & 0.74 & 0.60 \\
\hline White meat $(\mathrm{g} / \mathrm{d})$ & 38.34 & 32.02 & 47.34 & 42.68 & 0.12 & 0.54 \\
\hline Salt and salty snacks(g/d) & 36.79 & 38.78 & 41.18 & 47.54 & 0.51 & 0.99 \\
\hline Dairy $(g / d)$ & 274.24 & 234.54 & 301.84 & 214.28 & 0.39 & 0.70 \\
\hline Tea $(g / d)$ & 637.96 & 463.59 & 793.25 & 912.36 & 0.19 & 0.19 \\
\hline \multicolumn{7}{|l|}{ Dietary intake } \\
\hline Energy intake (kcal/d) & 2505.26 & 727.89 & 2615.62 & 754.66 & 0.31 & - \\
\hline Carbohydrate (g/d) & 362.65 & 126.42 & 370.55 & 117.74 & 0.65 & 0.20 \\
\hline Carbohydrate (\% energy) & 57.16 & 6.58 & 56.57 & 6.64 & 0.54 & 0.44 \\
\hline Protein $(\mathrm{g} / \mathrm{d})$ & 83.30 & 26.04 & 89.26 & 28.26 & 0.14 & 0.58 \\
\hline
\end{tabular}

MUFA, monounsaturated fatty acid; PUFA, polyunsaturated fatty acid; SFA, Saturated fatty acid

Data are presented as mean \pm standard deviation (SD).

${ }^{a}$ Calculated by analysis of variance and $p$-value $<0.05$ indicates a significant level.

*Obtained from Independent T-test

**Obtained from ANCOVA analysis

Food group adjusted by age, BMI, physical activity, and energy intake (Kcal)

Dietary intake (macronutrient and micronutrient) adjusted by energy intake (Kcal) 


\begin{tabular}{|c|c|c|c|c|c|c|}
\hline \multirow{3}{*}{$\begin{array}{l}\text { Variables } \\
\text { Protein (\% energy) }\end{array}$} & \multirow{2}{*}{\multicolumn{2}{|c|}{$\begin{array}{l}\text { Metabolic healthy } \\
\text { Mean SD }\end{array}$}} & \multirow{2}{*}{\multicolumn{2}{|c|}{$\begin{array}{l}\text { Metabolic unhealthy } \\
\text { Mean SD }\end{array}$}} & \multirow{3}{*}{$\begin{array}{l}\text { P-value* } \\
0.36\end{array}$} & \multirow{3}{*}{$\begin{array}{l}\text { P-value }^{\text {*ᄎ }} \\
0.64\end{array}$} \\
\hline & & & & & & \\
\hline & 13.43 & 2.61 & \multicolumn{2}{|c|}{$\begin{array}{l}\text { Mean SD } \\
1375 \quad 233\end{array}$} & & \\
\hline Fat $(\mathrm{g} / \mathrm{d})$ & 88.66 & 27.49 & 94.91 & 33.61 & 0.18 & 0.33 \\
\hline Fat (\% energy) & 32.38 & 5.79 & 32.75 & 6.66 & 0.82 & 0.62 \\
\hline MUFA (g/d) & 28.93 & 8.47 & 31.39 & 11.69 & 0.12 & 0.24 \\
\hline PUFA(g/d) & 18.84 & 6.71 & 20.12 & 9.06 & 0.30 & 0.64 \\
\hline $\operatorname{SFA}(g / d)$ & 25.99 & 9.76 & 28.43 & 11.60 & 0.13 & 0.14 \\
\hline Fiber $(g / d)$ & 45.18 & 20.05 & 44.30 & 17.86 & 0.74 & 0.15 \\
\hline \multicolumn{7}{|l|}{ Nutrients } \\
\hline Vitamin A (RAE) & 729.58 & 351.47 & 795.26 & 428.82 & 0.27 & 0.84 \\
\hline Thiamine $(\mathrm{mg} / \mathrm{d})$ & 2.01 & 0.65 & 2.05 & 0.63 & 0.63 & 0.39 \\
\hline Riboflavin (mg/d) & 2.05 & 0.71 & 2.23 & 0.84 & 0.14 & 0.30 \\
\hline Niacin $(\mathrm{mg} / \mathrm{d})$ & 23.59 & 7.09 & 25.25 & 9.30 & 0.19 & 0.74 \\
\hline Vitamin B6 (mg/d) & 2.06 & 0.67 & 2.17 & 0.72 & 0.28 & 0.80 \\
\hline Folic acid $(\mu \mathrm{g} / \mathrm{d})$ & 585.20 & 181.26 & 606.54 & 171.92 & 0.40 & 0.66 \\
\hline Vitamin B12 $(\mu \mathrm{g} / \mathrm{d})$ & 3.99 & 2.00 & 4.47 & 2.36 & 0.15 & 0.29 \\
\hline Vitamin C (mg/d) & 190.26 & 124.40 & 195.71 & 132.75 & 0.77 & 0.19 \\
\hline Vitamin E (mg/d) & 15.91 & 6.73 & 17.32 & 9.28 & 0.26 & 0.37 \\
\hline Vitamin $D(\mu \mathrm{g} / \mathrm{d})$ & 2.05 & 1.73 & 1.87 & 1.47 & 0.44 & 0.22 \\
\hline Iron $(\mathrm{mg} / \mathrm{d})$ & 18.28 & 6.03 & 18.63 & 5.90 & 0.68 & 0.14 \\
\hline
\end{tabular}

MUFA, monounsaturated fatty acid; PUFA, polyunsaturated fatty acid; SFA, Saturated fatty acid Data are presented as mean \pm standard deviation (SD).

a Calculated by analysis of variance and $p$-value $<0.05$ indicates a significant level.

*Obtained from Independent T-test

**Obtained from ANCOVA analysis

Food group adjusted by age, BMI, physical activity, and energy intake (Kcal)

Dietary intake (macronutrient and micronutrient) adjusted by energy intake (Kcal) 


\begin{tabular}{|c|c|c|c|c|c|c|}
\hline \multirow[t]{2}{*}{ Variables } & \multicolumn{2}{|c|}{ Metabolic healthy } & \multicolumn{2}{|c|}{ Metabolic unhealthy } & \multirow{2}{*}{ P-value* } & \multirow{2}{*}{ P-value } \\
\hline & \multicolumn{2}{|l|}{ Mean SD } & \multicolumn{2}{|l|}{ Mean SD } & & \\
\hline Selenium $(\mu \mathrm{g} / \mathrm{d})$ & 114.81 & 36.86 & 119.67 & 42.80 & 0.42 & 0.95 \\
\hline Zinc (mg/d) & 12.33 & 4.13 & 13.12 & 4.30 & 0.20 & 0.94 \\
\hline Magnesium $(\mathrm{mg} / \mathrm{d})$ & 443.71 & 144.98 & 463.73 & 150.39 & 0.36 & 0.65 \\
\hline Calcium (mg/d) & 1117.99 & 423.03 & 1158.55 & 410.52 & 0.50 & 0.82 \\
\hline Potassium (mg/d) & 4208.43 & 1662.90 & 4345.83 & 1538.23 & 0.55 & 0.44 \\
\hline Caffeine $(\mathrm{g} / \mathrm{d})$ & 147.13 & 105.55 & 157.53 & 178.52 & 0.66 & 0.72 \\
\hline \multicolumn{7}{|c|}{ MUFA, monounsaturated fatty acid; PUFA, polyunsaturated fatty acid; SFA, Saturated fatty acid } \\
\hline \multicolumn{7}{|c|}{ Data are presented as mean \pm standard deviation (SD). } \\
\hline \multicolumn{7}{|c|}{${ }^{a}$ Calculated by analysis of variance and $p$-value $<0.05$ indicates a significant level. } \\
\hline \multicolumn{7}{|c|}{ *Obtained from Independent T-test } \\
\hline \multicolumn{7}{|c|}{$\star \star$ Obtained from ANCOVA analysis } \\
\hline \multicolumn{7}{|c|}{ Food group adjusted by age, BMI, physical activity, and energy intake (Kcal) } \\
\hline Dietary intake (macr & and micre & hutrient) ac & usted by & rgy intak & Kcal) & \\
\hline
\end{tabular}

\section{Correlation of main variables with sarcopenic obesity}

Additional file 1: Table S1 contains the correlation between sarcopenic obesity with the variables of interest in this study. A simple correlation revealed a significant inverse associations between sarcopenic obesity with FFM $(\mathrm{r}=-0.30, p=<0.001)$ and FFMI $(\mathrm{r}=-0.12, p=0.01)$, while significant positive correlations were observed between sarcopenic obesity with BFM $(r=0.27, p=<0.001)$ and FMI $(r=0.33, p=<0.001)$.

\section{Association between obesity statuses with measures of body composition}

Association of SO with body composition are presented in Table 3. In the crude model, participants with MUO had 0.27 folds increased odds of FFM compared to than the MHO subjects $(p=0.001)$. However, after controlling for potential confounders (age, total energy intake, and physical activity level), the MUO groups had 0.29 folds decreased odds of FFM compared to the MHO group $(p=0.008)$. In the crude model, participants with MUO were observed to be positively associated with greater odds of increasing BFM than those with MHO phenotype $(p=0.01)$. The observed association between metabolic health statuses and BFM remained significant even after controlling for all potential confounders. Subjects with MUO phenotype in the crude model were observed to have a $0.87 \mathrm{~kg}$ lower SMM compared with the MHO subjects $(p=0.006)$. After age, total energy intake, and physical activity had been controlled for, the MUO 
participants had a 0.83 lower SMM compared with the MHO subjects $(p=0.01)$. Furthermore, after additional adjustment for weight and economic status, the association remained significant $(p=0.03)$. The control of all potential confounders revealed a 4.21 fold greater odds of sarcopenic obesity among MUO subjects $(p=0.02)$. 
Table 3

Association between metabolic healthy statuses with measures of body composition and sarcopenic obesity

$\begin{array}{llll}\text { Variables } & \beta & \text { OR }(95 \% \mathrm{Cl}) & \text { P-value }\end{array}$

\section{Fat-Free Mass}

\begin{tabular}{lllll} 
Crude & MUO & 0.76 & $0.27(0.13,0.56)$ & 0.001 \\
\hline MHO & Ref & Ref & \\
\hline MUO 1 & -0.89 & $0.29(0.13,0.62)$ & 0.008 \\
Model 2 & MHO & Ref & Ref & \\
& MUO & -0.55 & $0.26(0.11,0.59)$ & 0.07
\end{tabular}

\section{Body Fat Mass}

$\begin{array}{lllll}\text { Crude } & \text { MUO } & 1.31 & 3.73(1.27,10.91) & 0.01 \\ \text { Model 1 } & \text { MHO } & \text { Ref } & \text { Ref } & \\ & \text { MHO } & \text { Ref } & \text { Ref } & 0.01 \\ \text { Model 2 } & \text { MUO } & 0.95 & 2.60(1.39,17.20) & 0.02 \\ & \text { MHO } & \text { Ref } & \text { Ref } & \end{array}$

\section{Skeletal Muscle Mass}

\begin{tabular}{lllll} 
Crude & MUO & -0.87 & $0.50(0.27,0.90)$ & 0.006 \\
Model 1 & MHO & Ref & Ref & \\
& MUO & -0.83 & $0.46(0.22,0.84)$ & 0.01 \\
Model 2 & MHO & Ref & Ref & \\
\hline MUO & -0.73 & $0.47(0.24,0.94)$ & 0.03
\end{tabular}

MUO; metabolic unhealthy obese, $\mathrm{MH}$; metabolic obese

P-values are reported base on the Binary logistic regression test and are considered significant at $\llbracket 0.05$

Healthy obese is a reference group

Model 1: Adjusted for age, kcal, and physical activity

Model 2: Model 1 confounders with further adjustment with weight and economic status 


\begin{tabular}{|c|c|c|c|c|}
\hline \multirow[t]{2}{*}{ Variables } & & $\boldsymbol{\beta}$ & OR (95\% Cl) & P-value \\
\hline & MHO & Ref & Ref & \\
\hline \multicolumn{5}{|c|}{ Sarcopenic obesity } \\
\hline \multirow[t]{2}{*}{ Crude } & MUO & 0.35 & $1.42(0.45,4.45)$ & 0.54 \\
\hline & $\mathrm{MHO}$ & Ref & Ref & \\
\hline \multirow[t]{2}{*}{ Model 1} & MUO & 0.42 & $1.52(0.40,5.70)$ & 0.53 \\
\hline & $\mathrm{MHO}$ & Ref & Ref & \\
\hline \multirow[t]{2}{*}{ Model 2} & MUO & 1.43 & $4.21(1.28,62.26)$ & 0.02 \\
\hline & $\mathrm{MHO}$ & Ref & Ref & \\
\hline \multicolumn{5}{|c|}{ MUO; metabolic unhealthy obese, $\mathrm{MH}$; metabolic obese } \\
\hline \multicolumn{5}{|c|}{$\begin{array}{l}\text { P-values are reported base on the Binary logistic regression test and are considered significant at } \\
\bigotimes 0.05\end{array}$} \\
\hline \multicolumn{5}{|c|}{ Healthy obese is a reference group } \\
\hline \multicolumn{5}{|c|}{ Model 1: Adjusted for age, $\mathrm{kcal}$, and physical activity } \\
\hline
\end{tabular}

\section{Discussion}

This study was conducted among overweight and obese Iranian women between the ages of 18-50 years, with a $\mathrm{BMI} \geq 25 \mathrm{~kg} / \mathrm{m}^{2}$. The metabolic risk was assessed according to Karelis criteria and subjects classified as either MHO or MUO. Of the 241 subjects, $73.03 \%$ were classified as MUO with $7.88 \%$ as SO. We found that individuals with high FFM and high SMM to have a significantly low risk of MUO phenotype. We also found a significant positive correlation between sarcopenic obesity and MUO phenotype after all potential covariates were controlled.

This study's MUO phenotypes showed a significantly higher BFM, VFM, FFM, SMM, and FMI than the $\mathrm{MHO}$ group. Previous studies have found that MUO women had significantly higher BFM, VFM, and muscle mass than MHO women [21] and healthy obese people [29].

Despite the fact that some studies $[6,22,23]$ have observed a non-significant difference in the measures of adiposity in "healthy" and "unhealthy" phenotypes, in line with other previous studies [7, 24], we observed a significant increase in BFM in the MUO phenotype even after all potential confounders were controlled for. BFM has been reported as an independent predictor of insulin resistance and dyslipidemia [25] among postmenopausal women. Higher BFM has also been reported as an increased risk factor for type 2 diabetes [26, 27], as well as increase metabolic risk and the risk of cardiovascular disease [28, 29] 
in both gender. Furthermore, researches have shown increasing BFM to be correlated with an increase in FBG, triglyceride, LDL, and total cholesterol, and a decrease in HDL cholesterol [30, 31].

There have also been conflicting results regarding SMM accumulation among the metabolic phenotypes. While in some studies no significant differences in SMM indices between metabolic phenotypes of either obese or non-obese postmenopausal women [23] were found, some studies have reported SMM to significantly increase in the MUO phenotype of postmenopausal women [8, 32], and significantly decrease in the metabolic non-obese phenotype of young women (5). Estrella et al., [33] among Hispanic/Latino women found higher SMM to be independently associated with a lower prevalence of the MHO phenotype. In our study, each increase of 1-SD in skeletal muscle mass was associated with a lower prevalence of the MUO phenotype. Our result is in line with the fourth and fifth Korean National Health and Nutrition Examination Survey which reported a protective association of muscle mass with metabolic syndrome [34]. Additionally, in a 7-year retrospective cohort study, Kim and colleagues [35] found that an increase in relative skeletal muscle mass over time has a potential preventive effect on developing metabolic syndrome, independently of baseline skeletal muscle mass and glycometabolic parameters. In a nationally representative sample of 4,449 US adults aged 50 years and older from the NHANES surveys, Li and associates [36] found that only participants with low muscle mass but without metabolic syndrome had a significantly increased risk of all-cause mortality.

After we adjusted for all potential confounders, each increase of 1-SD in sarcopenic obesity was observed to be associated with a higher prevalence of MUO phenotype in the current study. In line with previous epidemiological studies, reports have shown that the odds of metabolic syndrome was 6 to 8 times higher in postmenopausal Korean women, elderly Korean men and women, and adult Caucasian subjects with sarcopenic obesity (SO) compared to those without sarcopenic obesity [14, 37-39]. Furthermore, Kim et al., [40] reported an increased risk of metabolic syndrome in subjects with sarcopenic obesity. In their study, compared with normal subjects, they found that SO subjects had significantly higher values for a number of metabolic syndrome components. Furthermore, they found $\mathrm{SO}$ to be independently associated with metabolic syndrome among women after adjustment for age.

\section{Conclusion}

In conclusion, increased adiposity and decreased skeletal muscle mass was associated with unfavorable metabolic traits among overweight and obese Iranian women. SO was also found to be associated with a greater risk of developing MUO phenotype after controlling for potential confounders.

\section{Limitations}

The cross-sectional nature of this study does not permit the assessment of causality. Also, we used BIA in order to assess body composition but not dual X-ray absorptiometry which is considered the gold standard method. However, BIA is also a validated and reliable method for the measurement of body 
composition [41]. Furthermore, the result of the current study cannot be generalized since only females were included in the study.

\section{Abbreviations}

BC body composition; BFM:body fat mass; BIA:Bioelectrical impedance analyzer BMl:body mass index; Cl:confidence intervals; DBP:diastolic blood pressure; DXA:dual X-ray absorptiometry; FBS:fasting blood sugar; FFM:fat-free mass; FFMl:fat-free mass index; FFQ:food frequency questionnaire; FM:fat mass; FMl:fat mass index; HC:hip circumference; HDL:high-density lipoprotein; HOMA-IR:homeostatic model assessment of insulin resistance; hs-CRP:high-sensitive C-reactive protein; IPAQ:International Physical Activity Questionnaire; LDL:Iow-density lipoprotein; MET:metabolic equivalent; MHO:metabolically healthy obese; MUO:metabolically unhealthy obese; OR:odds ratios; SBP:systolic blood pressure; SDs:standard deviations; SE:standard errors; SMM:skeletal muscle mass; SO:sarcopenic obesity; T-chol:Total cholesterol; TG triglyceride; TNF:tumor necrosis factor; VAT:visceral adipose tissue; WC:waist circumference; WHR:waist-to-hip ratio;

\section{Declarations}

\section{Ethical approval}

This study was approved by the ethics committee of Tehran University of Medical Sciences (TUMS) with the following identification IR.TUMS.VCR.REC.1398.692.

\section{Consent for publication}

Not applicable.

\section{Availability of data and materials}

Participants of this study did not agree for their data to be shared publicly, so supporting data is not available.

\section{Competing interests}

All authors declared that they have no competing interests

\section{Funding}

This study is funded by grants from the Tehran University of Medical Sciences (TUMS). (Grant ID: 97-03161-41155).

\section{Author's contributions}


ATJ wrote the article, AM and NR revised the article, FSH performed the statistical analyses, KhM had full access to all of the data in the study and took responsibility for the integrity and accuracy of the data. All authors read and approved the final manuscript.

\section{Acknowledgments}

The authors thank the study participants for their cooperation and assistance in physical examinations. This study was supported by TUMS.

\section{References}

1. Ser Ro.a.W.c.J.W.H.O.T.R., Obesity: preventing and managing the global epidemic. 2000. 894(i-xii): p. 1-253.

2. Wannamethee SG. Atkins. and J.L.J.P.o.t.N.S. Muscle loss obesity: the health implications of sarcopenia sarcopenic obesity. 2015;74(4):405-12.

3. Fallahzadeh $\mathrm{H}$, Saadati H, Keyghobadi NJSJ. Estimating the prevalence and trends of obesity in Iran populations from 2000 to 2011: a meta-analysis study. 2017. 25(9): p. 681-689.

4. Shojaei M, et al., Gender differences in the prevalence of cardiovascular risk factors in an Iranian urban population. 2015. 15(3): p. 178-184.

5. Vecchié A, et al., Obesity phenotypes and their paradoxical association with cardiovascular diseases. 2018. 48: p. 6-17.

6. Brochu M, et al., What are the physical characteristics associated with a normal metabolic profile despite a high level of obesity in postmenopausal women? 2001. 86(3): p. 1020-1025.

7. Karelis AD, et al., The metabolically healthy but obese individual presents a favorable inflammation profile. 2005. 90(7): p. 4145-4150.

8. Wildman RP, et al., The obese without cardiometabolic risk factor clustering and the normal weight with cardiometabolic risk factor clustering: prevalence and correlates of 2 phenotypes among the US population (NHANES 1999-2004). 2008. 168(15): p. 1617-1624.

9. Primeau $\mathrm{V}$, et al. Characterizing the profile of obese patients who are metabolically healthy. 2011;35(7):971-81.

10. Naukkarinen J, et al., Characterising metabolically healthy obesity in weight-discordant monozygotic twins. 2014. 57(1): p. 167-176.

11. Rosenberg, IJ.A.J.C.N.. Epidemiologic and methodologic problems in determining nutritional status of older persons.(Summary comments). 1989. 50: p. 1231-1233.

12. Baumgartner RN. J.A.o.t.N.Y.A.o.S. Body composition in healthy aging. 2000;904(1):437-48.

13. Wannamethee SG, et al., Decreased muscle mass and increased central adiposity are independently related to mortality in older men. 2007. 86(5): p. 1339-1346.

14. Lim S, et al., Sarcopenic obesity: prevalence and association with metabolic syndrome in the Korean Longitudinal Study on Health and Aging (KLoSHA). 2010. 33(7): p. 1652-1654. 
15. Chung J-Y, et al., Body composition and its association with cardiometabolic risk factors in the elderly: a focus on sarcopenic obesity. 2013. 56(1): p. 270-278.

16. Kim TN, et al., Association of low muscle mass and combined low muscle mass and visceral obesity with low cardiorespiratory fitness. 2014. 9(6): p. e100118.

17. Rasaei $\mathrm{N}$, et al., The association between sarcopenic obesity (SO) and major dietary patterns in overweight and obese adult women. 2019. 13(4): p. 2519-2524.

18. Rahmanian K, et al., Prevalence and clinical characteristics of metabolically unhealthy obesity in an Iranian adult population. 2019. 12: p. 1387.

19. Matthews D, et al., Turner RC. Homeostasis model assessment: insulin resistance and beta-cell function from fasting plasma glucose and insulin concentrations in man. 1985. 28(7): p. 412-9.

20. Hinnouho G-M, et al., Metabolically healthy obesity and risk of mortality: does the definition of metabolic health matter? 2013. 36(8): p. 2294-2300.

21. Chen L-K, et al. Sarcopenia in Asia: consensus report of the Asian Working. Group for Sarcopenia. 2014;15(2):95-101.

22. Messier V, et al., Metabolically healthy but obese individuals: relationship with hepatic enzymes. 2010. 59(1): p. 20-24.

23. Peppa M, et al., Body composition determinants of metabolic phenotypes of obesity in nonobese and obese postmenopausal women. 2013. 21(9): p. 1807-1814.

24. Kang EY, Yim J-EJNR, Practice. Differences in dietary intakes, body compositions, and biochemical indices between metabolically healthy and metabolically abnormal obese Korean women. 2019. 13(6): p. 488-497.

25. Van Pelt R, et al., Contributions of total and regional fat mass to risk for cardiovascular disease in older women. 2002.

26. Snijder MB, et al., Trunk fat and leg fat have independent and opposite associations with fasting and postload glucose levels: the Hoorn study. 2004. 27(2): p. 372-377.

27. Choi SI, et al., Relationship between regional body fat distribution and diabetes mellitus: 2008 to 2010 Korean National Health and Nutrition Examination Surveys. 2017. 41(1): p. 51-59.

28. Okura T, et al., Regional body composition changes exhibit opposing effects on coronary heart disease risk factors. 2004. 24(5): p. 923-929.

29. Madeira FB, et al., Normal weight obesity is associated with metabolic syndrome and insulin resistance in young adults from a middle-income country. 2013. 8(3): p. e60673.

30. Bi X, et al., DXA-measured visceral adipose tissue predicts impaired glucose tolerance and metabolic syndrome in obese Caucasian and African-American women. 2015. 69(3): p. 329-336.

31. Aucouturier J, et al., Effect of android to gynoid fat ratio on insulin resistance in obese youth. 2009. 163(9): p. 826-831.

32. Davies MJ, et al., Effects of moderate alcohol intake on fasting insulin and glucose concentrations and insulin sensitivity in postmenopausal women: a randomized controlled trial. 2002. 287(19): 
p. $2559-2562$.

33. Estrella ML, et al., Correlates of and Body Composition Measures Associated with metabolically healthy obesity phenotype in hispanic/latino women and men: the Hispanic Community Health Study/Study of Latinos (HCHS/SOL). 2019. 2019.

34. Kim K, Park SMJSr. Association of muscle mass and fat mass with insulin resistance and the prevalence of metabolic syndrome in Korean adults: a cross-sectional study. 2018. 8(1): p. 1-8.

35. Kim G, et al., Increase in relative skeletal muscle mass over time and its inverse association with metabolic syndrome development: a 7-year retrospective cohort study. 2018. 17(1): p. 1-13.

36. Li R, et al., Associations of muscle mass and strength with all-cause mortality among US older adults. 2018. 50(3): p. 458.

37. Kim TN, et al., Skeletal muscle mass to visceral fat area ratio is associated with metabolic syndrome and arterial stiffness: the Korean Sarcopenic Obesity Study (KSOS). 2011. 93(2): p. 285-291.

38. Kang S-Y, et al., Association between sarcopenic obesity and metabolic syndrome in postmenopausal women: a cross-sectional study based on the Korean National Health and Nutritional Examination Surveys from 2008 to 2011. 2017. 24(1): p. 9-14.

39. Poggiogalle E, et al., Sarcopenic obesity and metabolic syndrome in adult Caucasian subjects. 2016. 20(9): p. 958-963.

40. Kim TN, et al., Prevalence of sarcopenia and sarcopenic obesity in Korean adults: the Korean sarcopenic obesity study. 2009. 33(8): p. 885-892.

41. $\mathrm{Kim} \mathrm{H}$, et al., External cross-validation of bioelectrical impedance analysis for the assessment of body composition in Korean adults. 2011. 5(3): p. 246-252.

\section{Supplementary Files}

This is a list of supplementary files associated with this preprint. Click to download.

- AdditionalfileTableS1.docx 